\title{
Water is not a dynamic polydisperse branched polymer
}

Teresa Head-Gordon ${ }^{a, b, c, d, e, 1}$ and Francesco Paesani ${ }^{f, g, h}$

In PNAS, Naserifar and Goddard (1) report that their RexPoN water model under ambient conditions comprises a "dynamic polydisperse branched polymer," which they speculate explains the existence of the liquid-liquid critical point (LLCP) in the supercooled region. The observable they rely on to support this is the oxygen-oxygen radial distribution function, goo, from a dated neutron scattering experiment (1). Although it is well known that neutron scattering is almost exclusively sensitive to hydrogen correlations, and goo is more reliably obtained from X-ray scattering (2), they make the unsupported statement that "the most reliable goo curve is neutron where there is no inference from electrons" (1). However, two X-ray goo curves in figure $1 \mathrm{~B}$ of ref. 1 are from a joint neutron $X$-ray analysis (X-ray 1$)$ and neutron scattering study (X-ray3) (1). Given the importance that Naserifar and Goddard (1) place on goo, it is evident that their RexPoN model is in disagreement with the most reliable estimate of X-ray2 (2).

Naserifar and Goddard (1) dismiss the MB-pol model as inaccurate because the calculated coordination number from $g_{0 O}$ is too high at $\mathrm{N}_{\mathrm{OO}}=6.2$. MB-pol, using path integral molecular dynamics (3), yields $\mathrm{N}_{\mathrm{OO}}=4.8$, in good agreement with the experimental estimate of $\mathrm{N}_{\mathrm{OO}}=4.5$. Since RexPoN is fitted from quantum mechanics (1), the fact that the reported properties are based on classical molecular dynamics simulations (1) means that the necessary inclusion of nuclear quantum effects (4) will diminish its apparent agreement with experiment to yield a far less accurate model than claimed for other water properties, but especially for the liquid structure that is the lynchpin of their claims of superiority of RexPoN.
Naserifar and Goddard state that the RexPoN simulation melts from 4-coordinated ice into an ambient liquid with 2 strong hydrogen bonds (SHBs) that couple water molecules into isolatable regions resembling a branched nanopolymer. More specifically, they observe that SHBs "connect to form multibranched polymer chains $\left(151 \mathrm{H}_{2} \mathrm{O}\right.$ per chain at $298 \mathrm{~K}$ ), where branch points have $3 \mathrm{SHBs}$ and termination points have $1 \mathrm{SHB} . "$ They then speculate using this "structural feature" for the existence of the LLCP at $227 \mathrm{~K}$ and 1 bar (1).

There are several factual errors and speculation in Naserifar and Goddard's paper (1) on this point. Ambient water characterized by vibrational Raman and X-ray spectroscopies $(5,6)$ is explained by a homogeneous liquid state without any unusual structural motifs $(7,8)$. Even if a second critical point for supercooled water exists, it is irrelevant at $298 \mathrm{~K}$ since above a critical point, matter is homogeneous and should not have isolatable structural motifs. Finally, recent work by Kim et al. (9) has reported a compressibility maximum at $227 \mathrm{~K}$ and 1 bar, which is consistent with (but does not prove) an LLCP at likely a higher pressure and lower temperature. Well-validated models such as MB-pol (3) and IAMOEBA (10) are reasonably accurate in the supercooled region for the structure factor and the isothermal compressibility to analyze experiments that support the LLCP (9). However, these water models do not yield an ambient liquid with discernable structural domains, dynamic or not, but are simply described as a deformable tetrahedral hydrogenbonded network above a percolation threshold in which every water molecule experiences the same intermolecular force on average.

1 S. Naserifar, W. A. Goddard 3rd, Liquid water is a dynamic polydisperse branched polymer. Proc. Natl. Acad. Sci. U.S.A. 116, 19982003 (2019).

2 L. B. Skinner et al., Benchmark oxygen-oxygen pair-distribution function of ambient water from x-ray diffraction measurements with a wide Q-range. J. Chem. Phys. 138, 074506 (2013).

\footnotetext{
${ }^{a}$ Pitzer Center for Theoretical Chemistry, Berkeley, CA 94720; b Department of Chemistry, University of California, Berkeley, CA 94720; ' Department of Bioengineering, University of California, Berkeley, CA 94720; 'Department of Chemical and Biomolecular Engineering, University of California, Berkeley, CA 94720; ' Chemical Sciences Division, Lawrence Berkeley National Laboratory, Berkeley, CA 94720; 'Department of Chemistry and Biochemistry, University of California, San Diego, La Jolla, CA 92093; 9 Materials Science and Engineering, University of California, San Diego, La Jolla, CA 92093; and ' San Diego Supercomputer Center, University of California, San Diego, La Jolla, CA 92093

Author contributions: T.H.-G. and F.P. designed research; T.H.-G. and F.P. performed research; T.H.-G. and F.P. analyzed data; and T.H.-G. wrote the paper.

The authors declare no conflict of interest.

Published under the PNAS license.

${ }^{1}$ To whom correspondence may be addressed. Email: thg@berkeley.edu.

Published online June 25, 2019.
} 
3 G. R. Medders, V. Babin, F. Paesani, Development of a "first-principles" water potential with flexible monomers. III. Liquid phase properties. J. Chem. Theory Comput. 10, 2906-2910 (2014).

4 T. E. Markland, T. E. M. Ceriotti, Nuclear quantum effects enter the mainstream. Nat. Rev. Chem. 2, 0109 (2018).

5 G. W. Robinson, C. H. Cho, J. Urquidi, Isosbestic points in liquid water: Further strong evidence for the two-state mixture model. J. Chem. Phys. 111, 698-702 (1999).

6 C. Huang et al., The inhomogeneous structure of water at ambient conditions. Proc. Natl. Acad. Sci. U.S.A. 106, 15214-15218 (2009).

7 J. D. Smith et al., Unified description of temperature-dependent hydrogen-bond rearrangements in liquid water. Proc. Natl. Acad. Sci. U.S.A. 102, 14171-14174 (2005).

8 G. N. Clark, G. L. Hura, J. Teixeira, A. K. Soper, T. Head-Gordon, Small-angle scattering and the structure of ambient liquid water. Proc. Natl. Acad. Sci. U.S.A. 107, 14003-14007 (2010).

9 K. H. Kim et al., Maxima in the thermodynamic response and correlation functions of deeply supercooled water. Science 358, 1589-1593 (2017).

10 L. P. Wang et al., Systematic improvement of a classical molecular model of water. J. Phys. Chem. B 117, 9956-9972 (2013). 